\title{
Correction to: Differential Immunomodulatory Effect of Carbon Dots Influenced by the Type of Surface Passivation Agent
}

\author{
Furkan Ayaz, ${ }^{1,3}$ Melis Ozge Alas, ${ }^{2}$ and Rukan Genc ${ }^{2,3}$
}

Correction to: Inflammation (2019)

https://doi.org/10.1007/s10753-019-01165-0

In the Published article, the article title shows "Differential Immunomodulatory Effect of Carbon Dots Influenced". It should be "Differential Immunomodulatory Effect of Carbon Dots Influenced by the Type of Surface Passivation Agent".

The original article has been corrected.

The online version of the original article can be found at https://doi.org/ 10.1007/s10753-019-01165-0

\footnotetext{
${ }^{1}$ Department of Biotechnology, Faculty of Arts and Science, Mersin University, 33110 Mersin, Turkey

${ }^{2}$ Department of Chemical Engineering, Faculty of Engineering, Mersin University, 33110 Mersin, Turkey

${ }^{3}$ To whom correspondence should be addressed to Furkan Ayaz at Department of Biotechnology, Faculty of Arts and Science, Mersin University, 33110 Mersin, Turkey. E-mail: furkanayaz@mersin.edu.tr; and Rukan Genc at Department of Chemical Engineering, Faculty of Engineering, Mersin University, 33110 Mersin, Turkey. E-mail: rgenc@mersin.edu.tr
} 